\title{
Acompanhamento do estado nutricional e consumo alimentar de idosos durante o período de internação hospitalar
}

\author{
Ana Paula Trentin*, Juliana Rombaldi Bernardi**, Josiane Siviero***
}

\section{Resumo}

O objetivo deste estudo foi acompanhar o estado nutricional e o consumo alimentar de idosos durante o período de internação hospitalar no hospital do município de Guaporé, Rio Grande do Sul. Trata-se de um estudo de coorte, com a utilização de dados primários, realizado em 2014-2015. A amostra foi composta por 62 idosos. Por meio de formulário, obtiveram-se os dados, como: gênero, idade, doença e tipo de dieta. Aplicou-se a Mini Avaliação Nutricional e o Recordatório Alimentar de 24 horas, no qual se avaliou o total de calorias, carboidrato, proteína, lipídeos, fibras, cálcio e ferro. Ambos foram aplicados no momento da internação, dentro de 24 horas, e posteriormente, na alta hospitalar. A análise estatística foi realizada com o programa Statistical Package for Social Sciences. As variáveis quantitativas foram descritas por média e desvio padrão ou mediana e amplitude interquartílica e as qualitativas por frequências absolutas e relativas. Os resultados demonstraram que $21 \%$ dos idosos apresentaram magreza, 25,8\% eutrofia e $53,2 \%$ excesso de peso, sendo que não houve mudanças significativas em re- lação às medidas antropométricas durante o período de internação. Verificou-se que houve aumento significativo do consumo alimentar da internação para um dia pré-alta hospitalar em praticamente todos os nutrientes, exceto para o percentual de proteínas e para o de lipídeos, onde houve redução significativa na segunda avaliação. Encontrou-se elevada prevalência de excesso de peso na amostra, sem perda de peso nos dias de internação hospitalar, mas com melhora na ingestão alimentar para calorias e a maioria dos nutrientes.

Palavras chave: Estado Nutricional. Internação Hospitalar. Ingestão Alimentar. Idosos

\section{Introdução}

A população idosa brasileira vem crescendo de maneira significativa nas últimas cinco décadas, isso vem ocorrendo devido às transições decorrentes de mudanças nas taxas de mortalidade e de

* Nutricionista, Faculdade Cenecista de Bento Gonçalves (CNEC). Endereço para correspondência: Rua Palmira Pandolfo, nº 215, Bairro Planalto, Guaporé-RS. Telefone: (54) 98165 2250. E-mail: ana_trentin@ yahoo.com.br.

** Nutricionista, Doutora em Saúde da Criança e do Adolescente pela Universidade Federal do Rio Grande do Sul (Ufrgs); Mestre em Saúde da Criança e do Adolescente pela Universidade Federal de Ciências da Saúde de Porto Alegre (UFCSPA). Docente do Curso de Nutrição da Universidade Federal do Rio Grande do Sul (Ufrgs). Email: juliana.bernardi@yahoo.com.br

**** Nutricionista, Doutora em Ciências da Saúde pela Pontifícia Universidade Católica do Rio Grande do Sul (Pucrs); Mestre em Gerontologia Biomédica pelo Instituto de Geriatria e Gerontologia IGG/Pucrs. Docente do Curso de Nutrição da Faculdade Cenecista de Bento Gonçalves (CNEC) e da Universidade de Caxias do Sul (UCS).: Email: josianesiviero@hotmail.com

$\rightarrow$ http://dx.doi.org/10.5335/rbceh.v13i2.5543

Recebido em: 26.11.2015. Aceito em: 09.11.2016. 
fecundidade (INSTITUTO BRASILEIRO DE GEOGRAFIA E ESTATÍSTICA, 2010), sendo a saúde dessa população, um desafio e uma prioridade da saúde pública moderna (PAGANOTTO; SILVEIRA; VELASCO, 2012).

De acordo com relatório publicado em Genebra, estima-se que o número de pessoas idosas duplique até o ano de 2050 (ORGANIZAÇÃO MUNDIAL DA SAÚDE, 2015). Devido a isso, um dos aspectos observados no envelhecimento populacional acelerado foi o crescimento do número de idosos institucionalizados, que são acometidos por diferentes tipos de alterações metabólicas, fisiológicas, anatômicas e psicossociais inerentes à idade, em que, do ponto de vista nutricional, são considerados vulneráveis. Esse desequilíbrio nutricional nos idosos está reconhecidamente relacionado a um aumento da mortalidade, à susceptibilidade a infecções e à redução da qualidade de vida (FÉLIX; SOUZA, 2009).

Segundo a Organização Mundial da Saúde, a desnutrição ou má nutrição é o estado nutricional do indivíduo caracterizado pela baixa ingestão de energia, micronutrientes e proteína que resulta de complexa interação entre sua alimentação, condições econômicas, estado de saúde e condições sociais em que vive (AQUINO, 2005). Norman et al. (2008) afirmam que a desnutrição afeta adversamente a evolução clínica de pacientes hospitalizados, prolongando o tempo de permanência e os custos hospitalares. Adicionalmente, estudo de revisão da literatura com o objetivo de analisar os fatores associados à desnutrição em idosos institucionalizados encontrou que os fatores psicológicos (depressão e demência) e funcionais (dependência) foram os principais aspectos relacionados à desnutrição, podendo comprometer a qualidade de vida do idoso (SILVA et al., 2015).

Além disso, o estudo de Nascimento et al. (2011) cita que, dentre as condições associadas às alterações do estado nutricional, destacam-se a hipertensão arterial, o diabetes mellitus, as dislipidemias, a artrite, a polifarmácia e o maior número de internações. Os autores evidenciaram em seus resultados um cenário caracterizado pela prevalência elevada de excesso de peso, entre as mulheres e entre os que relataram artrite/artrose. $\mathrm{E}$, ao contrário, encontraram baixo peso entre os homens, especialmente associado aos que relataram pior percepção da saúde. Apontam, ainda, que os idosos são mais vulneráveis ao baixo peso devido ao processo de envelhecimento que está relacionado a mudanças da composição corporal, ficando, assim, mais propensos aos riscos decorrentes da perda de peso. Nesse sentido, a avaliação do estado nutricional dos pacientes hospitalizados é fundamental na medida em que se reconhece a alta prevalência de risco nutricional neste grupo (CRESTANI et al., 2011).

Waitzberg, Caiefee e Correia (1999) ressaltam que diante do panorama da desnutrição no Brasil, torna-se clara a importância de se estabelecer o diagnóstico nutricional por meio de ferramentas e recursos que estão disponíveis atualmente, dentro dos próprios hospitais. 
Entre essas, pode-se citar a avaliação nutricional e o uso de terapia nutricional. Para Kruizenga et al. (2005), avaliar o estado nutricional é importante para que as medidas possam ser aplicadas na prevenção e no tratamento da desnutrição.

De acordo com Garcia (2006), entre os fatores causais atribuídos à desnutrição hospitalar, a alimentação é considerada um fator circunstancial em razão das mudanças alimentares e da troca de hábitos e horários das refeições. São diversas as situações clínicas que podem causar diminuição do apetite ou que podem dificultar a ingestão alimentar, além disso, alguns procedimentos de investigação e/ou de intervenção podem acarretar a necessidade constante de jejum ou alterações na dieta (HORNBY et al., 2005).

Em estudo realizado por Merhi et al. (2015) avaliando a redução de peso corporal, a dieta prescrita e a aceitação dos alimentos durante a internação hospitalar em 456 pacientes adultos e idosos $(38,8 \%)$ hospitalizados, observou-se que houve mudanças positivas na aceitação da dieta hospitalar. Os autores encontraram que houve melhora da aceitação da dieta do $1^{\circ}$ ao $3^{\circ}$ dia de internação, entretanto, ocorreu perda de peso significativa, relacionada ao sexo e ao tipo de doença avaliada. Ressalta-se a importância de tais investigações, pois a perda de peso pode comprometer a evolução clínica do paciente em diversas situações.

Sendo assim, com base no exposto acima, o objetivo deste estudo foi acompanhar o estado nutricional e o consumo alimentar de idosos durante o período de internação em um hospital no município de Guaporé, RS.

\section{Metodologia}

Trata-se de um estudo de coorte, com a utilização de dados primários, realizado no hospital da cidade de Guaporé, RS, no período de agosto de 2014 a julho de 2015. Este estudo teve aprovação pelo Comitê de Ética e Pesquisa da Faculdade Cenecista de Bento Gonçalves, sob protocolo número 717.134. Todos os participantes voluntários e/ou responsáveis assinaram o Termo de Consentimento Livre e Esclarecido (TCLE).

Os critérios de inclusão determinados para o estudo foram: idosos acima de 60 anos, de ambos os gêneros, internados no hospital por um período superior ou igual a cinco dias. Os critérios de exclusão foram idosos acamados sem mobilização, em uso de Terapia Nutricional Enteral, com distúrbios neurológicos, apresentando edema, aqueles que se recusaram a participar de alguma etapa do estudo e aqueles que permaneceram internados por um período inferior a cinco dias.

Conforme cálculo amostral, realizado com auxílio do programa Epi Info versão 3.5.2, estimou-se que, para a prevalência de $40,2 \%$ de desnutrição em idosos hospitalizados (VIDAL et al., 2008), com um nível de confiança de $95 \%$ e margem de erro de $8 \%$ e perdas amostrais de $5 \%$, o resultado deste cálculo estatístico foi de 151 idosos. A seleção da amostra deste estudo foi construída aleatoriamente 
de forma randômica. No período determinado para a coleta de dados foram avaliados no momento da internação 105 idosos, porém, desses, 43 receberam alta antes dos cinco dias, período estipulado como critério de inclusão no presente estudo, sendo avaliados 62 idosos no total.

Obtiveram-se as informações sobre: gênero, idade (anos), doença e o tipo de dieta, sendo questionadas ao próprio paciente e/ou confirmadas por meio do prontuário com registro médico e dos profissionais envolvidos nos atendimentos. Além disso, aplicou-se a Mini Avaliação Nutricional (MAN) e o Recordatório Alimentar de 24 horas (R24h). Ambos foram realizados no momento da internação, dentro de 24 horas e, posteriormente, na alta hospitalar.

Utilizou-se o questionário da Mini Avaliação Nutricional (GUIGOZ et al., 1996) para a determinação do estado nutricional, sendo o mesmo dividido, além da triagem nutricional, em quatro partes: avaliação antropométrica (índice de massa corporal (IMC), circunferência do braço, circunferência da panturrilha e perda de peso); avaliação global (perguntas relacionadas com o modo de vida, medicação, mobilidade e problemas psicológicos); avaliação dietética (perguntas relativas ao número de refeições, ingestão de alimentos e líquidos e autonomia na alimentação); e a autoavaliação (a auto percepção da saúde e da condição nutricional) (MIRANDA et al., 2012).

$\mathrm{O}$ peso foi aferido em uma balança mecânica portátil de marca G-tech®, com antiderrapante, previamente calibrada, com capacidade de até $150 \mathrm{~kg}$ (quilos) e precisão de 100 gramas. Foi solicitado que o idoso ficasse descalço, com roupas leves, posicionado ereto, com os calcanhares juntos no centro da balança, e os braços esticados ao lado do corpo (KAMIMURA et al., 2005). Após a estabilização do marcador, foi registrado o resultado da medida. Para obtenção da altura, foi utilizado o estadiômetro portátil da marca Sanny® com escala em milímetros, disposto em superfície firme e plana. Solicitou-se que o idoso ficasse posicionado em pé, descalço, com os calcanhares juntos e a cabeça no plano de Frankfurt (KAMIMURA et al., 2005). Com essas duas medidas, foi calculado o IMC. O cálculo foi realizado por meio da divisão da massa corporal $(\mathrm{kg})$ pela estatura (metros) ao quadrado. Em seguida, foi realizada classificação segundo Lipschitz (1994), em que o IMC $<22 \mathrm{~kg} /$ $\mathrm{m}^{2}$ classifica-se como magreza, de 22 a 27 eutrofia e $>27$, o idoso é classificado com excesso de peso.

Para aferição da circunferência do braço (CB) e da circunferência da panturrilha $(\mathrm{CP})$, foi utilizado uma fita métrica inelástica de $150 \mathrm{~cm}$, com precisão de 0,1 $\mathrm{cm}$. Na aferição da circinferência do braço, o idoso permaneceu com o braço extendido e relaxado ao longo do corpo, foi aferida a circunferência do ponto médio entre o processo acromial da escápula e o olecrano da ulna (FRISANCHO, 1990). A determinação do estado nutricional, conforme Nhanes III (AMERICAN HEART ASSOCIATION, 1996), foi por meio da classificação em percentis sendo: $(<\mathrm{P} 10)$ desnutrido, $(\mathrm{P} 10-\mathrm{P} 25)$ risco nutricional, (P25 - P75) eutrofia, (>P75 - <P85) risco 
de sobrepeso, (>P85 - <P90) sobrepeso e (>P90) obesidade.

Quanto à aferição da circunferência da panturilha, o idoso ficou em posição supina, com o joelho dobrado em um ângulo de $90^{\circ} \mathrm{C}$, com o calcanhar apoiado na cama ou na cadeira, sendo medida a maior circunferência da panturilha (OMS, 1995) e a determinação do estado nutricional em $<31 \mathrm{~cm}$ estavam em risco nutricional $\mathrm{e}>31 \mathrm{~cm}$ adequado (sem risco nutricional).

A obtenção do consumo alimentar foi realizada por meio do Recordatório Alimentar de 24 horas (R24h), no qual o idoso relatou o tipo e a quantidade de alimentos e líquidos ingeridos no período de 24 horas (1 dia), descrevendo, também, os horários das refeições. O tamanho das porções foi estimado pela demonstração das medidas caseiras (talheres, xícaras, prato e copo) ao paciente no momento da entrevista. Para o cálculo dos R24h foi utilizado o Programa Avanutri ${ }^{\circledR}$ 4.0. Avaliou-se o total de calorias e dentre os nutrientes selecionados para este estudo foram carboidrato $(\mathrm{g})$, proteína (g), lipídeos (g), fibras (g), cálcio (mg) e ferro (mg).

Os dados coletados foram, inicialmente, digitados em planilha excell ${ }^{\circledR}$ e, posteriormente, a análise estatística feita com o apoio do programa estatístico Statistical Package for Social Sciences (SPSS®) versão 21.0. As variáveis quantitativas foram descritas por média e desvio padrão ou mediana e amplitude interquartílica. As variáveis qualitativas foram descritas por frequências absolutas e relativas. Para comparar médias entre as duas avaliações durante o período de internação hospitalar, o teste $t$-student para amostras pareadas foi aplicado. Em caso de assimetria, o teste de Wilcoxon foi utilizado. Na comparação de proporções, o teste Qui-Quadrado de McNemar foi aplicado. Nas associações das variações de peso com idade e tempo de internação, os coeficientes de correlação de Pearson ou Spearman foram aplicados. Na comparação da variação média de peso entre os tipos de dieta, gênero e patologias, o teste $t$-student para amostras independentes foi aplicado. $\mathrm{O}$ nível de significância adotado foi de $5 \%$ $(\mathrm{p} \leq 0,05)$.

\section{Resultados}

Dos 62 pacientes avaliados, a idade média foi de $73,5 \pm 9,0$ anos, sendo que apenas $25,8 \%$ (16) tinham idade superior a 80 anos. Ainda conforme a tabela 1, a amostra foi composta por $48,4 \%$ de idosos do gênero masculino e $51,6 \%$ do gênero feminino. Em relação às patologias, $62,9 \%$ (39) possuíam doenças respiratórias, seguido de $24,2 \%$ (15) com Hipertensão Arterial Sistêmica (HAS). Em relação aos tipos de dieta, a maioria dos idosos $(38,7 \%)$ estava com alimentação livre. 
Tabela 1 - Caracterização da amostra de idosos hospitalizados

\begin{tabular}{|c|c|}
\hline Variáveis & $n=62$ \\
\hline Idade (anos) - média \pm DP & $73,5 \pm 9,0$ \\
\hline \multicolumn{2}{|l|}{ Faixa etária $-\mathrm{n}(\%)$} \\
\hline $60-69$ anos & $25(40,3)$ \\
\hline $70-79$ anos & $21(33,9)$ \\
\hline$\geq 80$ anos & $16(25,8)$ \\
\hline \multicolumn{2}{|l|}{ Sexo $-n(\%)$} \\
\hline Masculino & $30(48,4)$ \\
\hline Feminino & $32(51,6)$ \\
\hline \multicolumn{2}{|l|}{ Patologias - n(\%) } \\
\hline $\begin{array}{l}\text { Distúrbios respiratórios (DPOC, insuficiência respiratória, enfise- } \\
\text { ma pulmonar, pneumonia, tosse) }\end{array}$ & $39(62,9)$ \\
\hline ICC & $10(16,1)$ \\
\hline HAS & $15(24,2)$ \\
\hline Insuf. renal, lítiase renal, gota & $3(4,8)$ \\
\hline DM & $6(9,7)$ \\
\hline Câncer & $3(4,8)$ \\
\hline Outras (Nevralgia Facial, Anemia, etc.) & $17(27,4)$ \\
\hline Dias de internação - média \pm DP [min - max] & $6,8 \pm 1,2[5-10]$ \\
\hline \multicolumn{2}{|l|}{ Tipo de dieta $-\mathrm{n}(\%)$} \\
\hline Livre & $24(38,7)$ \\
\hline Branda & $17(27,4)$ \\
\hline Hipossódica & $21(33,9)$ \\
\hline Para diabéticos & $6(9,7)$ \\
\hline Hipercalórica e hiperproteica & $1(1,6)$ \\
\hline Dieta para insuficiência renal & $1(1,6)$ \\
\hline Líquida e pastosa & $1(1,6)$ \\
\hline
\end{tabular}

Fonte: primária.

Nota: $\mathrm{n}=$ número; \%= percentual; $\mathrm{DP}=$ desvio padrão; $\pm=$ mais ou menos; $\geq$ maior ou igual; min= mínimo; máx.= máximo; DPOC= Doença Pulmonar Obstrutiva Crônica; ICC= Insuficiência Cardíaca Congestiva; HAS= Hipertensão Arterial Sistêmica; DM= Diabetes Mellitus; etc $=$ e outras coisas.

Segundo descrito na Tabela 2, verificou-se que houve aumento significativo do consumo alimentar da internação para um dia pré-alta hospitalar em praticamente todos os nutrientes (calorias totais, carboidratos, fibras, cálcio e ferro; $\mathrm{p}<0,05)$, exceto para o percentual de proteínas $(\mathrm{p}=0,664)$. Para o percentual de lipídeos, houve redução significativa da ingestão na segunda avaliação $(\mathrm{p}=0,026)$. 
Tabela 2 - Avaliação da ingestão energética dos nutrientes nas avaliações de internação e pré-alta hospitalar

\begin{tabular}{l|c|c|c}
\hline \multirow{2}{*}{ Variáveis } & Internação hospitalar & Pré-alta hospitalar & \multirow{2}{*}{$\mathrm{P}$} \\
\cline { 2 - 3 } & Md (P25-P75) & Md (P25-P75) & $<0,001$ \\
Calorias & $859(618-1274)$ & $1424(1265-1630)$ & 0,011 \\
CHO (\%) & $60,5(53,1-68,6)$ & $65,8(62,6-69,0)$ & 0,664 \\
PTN (\%) & $14,0(11,7-19)$ & $13,7(12,8-16,8)$ & 0,026 \\
LIP (\%) & $23,4(16,5-28,4)$ & $18,8(16,6-22,5)$ & $<0,001$ \\
Fibras (g) & $5,0(2,3-9,7)$ & $9,6(8,2-12,4)$ & 0,012 \\
Ca (mg) & $127(70-203)$ & $184(139-234)$ & $<0,001$ \\
Fe (mg) & $5,4(3,1-7,7)$ & $8,6(6,8-9,9)$ & \\
\hline
\end{tabular}

Fonte: primária.

Nota: $\mathrm{g}=$ gramas; $\mathrm{mg}=$ miligramas; $\mathrm{md}=$ mediana; $\mathrm{p}=$ percentil; $\%=$ percentual $<=$ menor que CHO= carboidrato; $\mathrm{PTN}=$ proteína; LIP= lipídeos; $\mathrm{Ca}=$ cálcio; Fe ferro.

* teste de Wilcoxon.

Conforme informações apresentadas na tabela 3 , o percentual de três refeições completas $(p=0,002)$, três ingestões de proteínas $(\mathrm{p}<0,001)$, duas ou mais frutas ou verduras/dia $(p=0,031)$ e três ou mais xícaras de líquidos/dia $(p=0,050)$ aumentou significativamente comparando-se o período de internação ao de pré-alta hospitalar. No entanto, o risco nutricional, avaliado pelo escore total do MAN, não teve modificações estatisticamente significativas $(p>0,05)$.

Quanto à ingestão de líquidos, o aumento se deu, principalmente, nos pacientes que ingeriram baixa quantidade de água inferior a de 3 xícaras por dia $(p=0,050)$.

Tabela 3 - Avaliação do risco nutricional, pela MAN, nas avaliações de internação e pré-alta hospitalar

Continua

\begin{tabular}{|c|c|c|c|}
\hline \multirow[t]{2}{*}{ Questões } & $\begin{array}{l}\text { Internação } \\
\text { hospitalar }\end{array}$ & $\begin{array}{l}\text { Pré-alta } \\
\text { hospitalar }\end{array}$ & \multirow[t]{2}{*}{$\mathrm{p}^{*}$} \\
\hline & $\mathrm{n}(\%)$ & $\mathrm{n}(\%)$ & \\
\hline $\begin{array}{l}\text { 1. Menor ingestão alimentar } \\
\text { Perda de apetite severa } \\
\text { Perda de apetite moderada } \\
\text { Nenhuma perda de apetite }\end{array}$ & $\begin{array}{r}3(4,8) \\
34(54,8) \\
25(40,3)\end{array}$ & $\begin{array}{r}3(4,8) \\
33(53,2) \\
26(41,9)\end{array}$ & 0,317 \\
\hline $\begin{array}{l}\text { 2. } \quad \text { Perda peso nos últimos 3meses } \\
\text { Superior a } 3 \mathrm{~kg} \\
\text { Não sabe } \\
\text { Entre } 1 \text { e } 3 \mathrm{~kg} \\
\text { Nenhuma perda } \\
\end{array}$ & $\begin{array}{r}16(25,8) \\
3(4,8) \\
16(25,8) \\
27(43,5)\end{array}$ & $\begin{array}{r}16(25,8) \\
2(3,2) \\
17(27,4) \\
27(43,5)\end{array}$ & 0,317 \\
\hline $\begin{array}{l}\text { 3. Mobilidade } \\
\text { Pode sair, mas não sai } \\
\text { Sai }\end{array}$ & $\begin{array}{r}5(8,1) \\
57(91,9)\end{array}$ & $\begin{array}{r}5(8,1) \\
57(91,9)\end{array}$ & 1,000 \\
\hline $\begin{array}{l}\text { 4. Estresse psicológico ou doença aguda nos últimos 3m } \\
\text { Sim } \\
\text { Não }\end{array}$ & $\begin{array}{l}22(35,5) \\
40(64,5) \\
\end{array}$ & $\begin{array}{l}22(35,5) \\
40(64,5)\end{array}$ & 1,000 \\
\hline $\begin{array}{l}\text { 5. Problemas neuropsicológicos } \\
\text { Demência severa ou depressão } \\
\text { Sem problemas psicológicos }\end{array}$ & $\begin{array}{l}21(33,9) \\
41(66,1) \\
\end{array}$ & $\begin{array}{l}22(35,5) \\
40(64,5)\end{array}$ & 1,000 \\
\hline
\end{tabular}


Conclusão

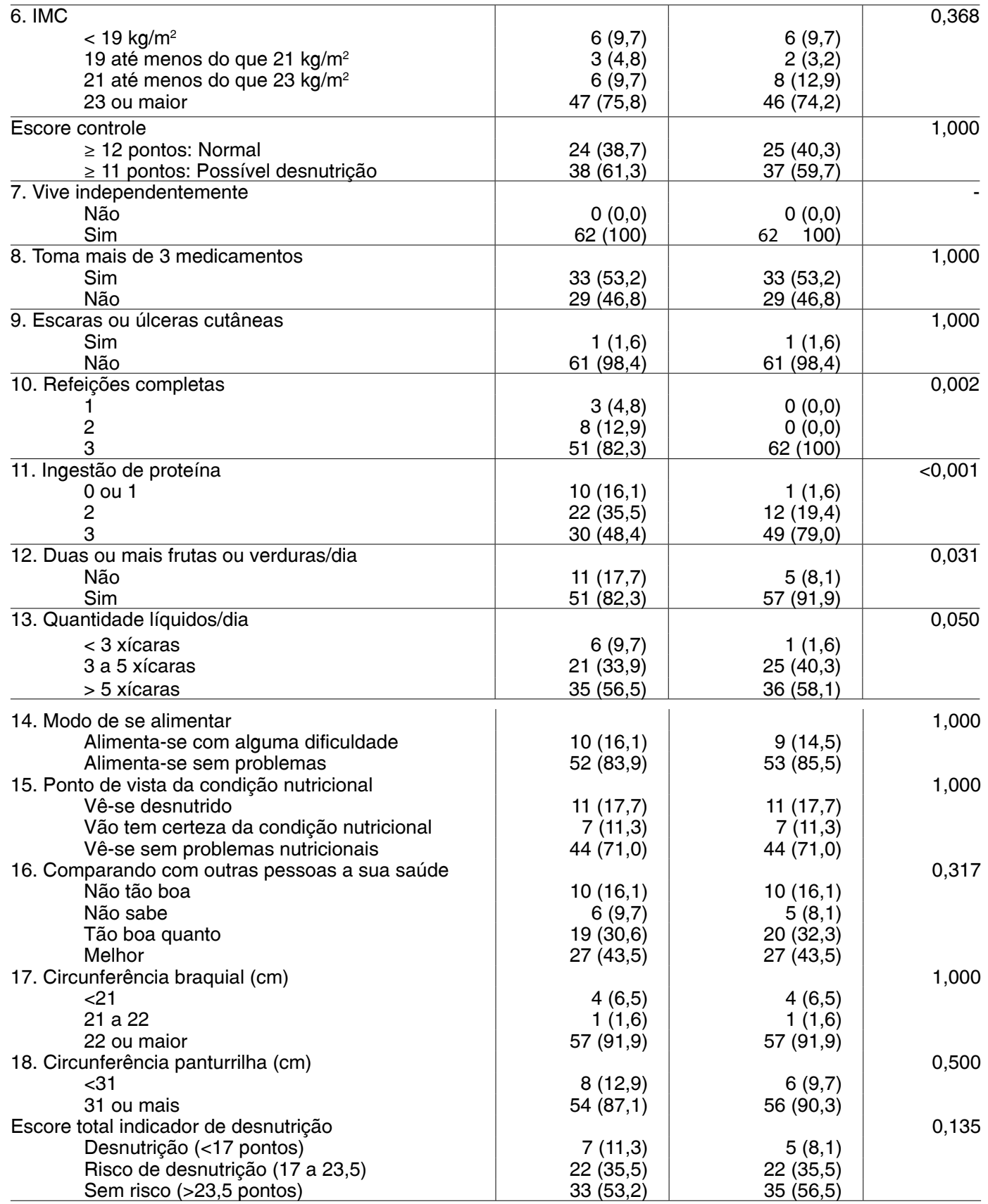

Fonte: primária.

Nota: $\mathrm{Md}=$ média; $\mathrm{p}=$ percentil; $\mathrm{n}=$ número; $\%=$ percentual; $\mathrm{m}=$ meses; $\mathrm{kg}=$ quilos; $<=$ menor que; $\mathrm{kg} / \mathrm{m}^{2}=$ quilos por metro quadrado; $\geq=$ maior ou igual; >= maior do que; IMC: índice de massa corporal.

* teste qui-quadrado de McNemar. 
Não houve mudança significativa nos dados antropométricos no período de internação hospitalar, de acordo com os dados apresentados na Tabela 4 . Não houve associação significativa com nenhuma das variáveis ( $\mathrm{p}>0,05)$, muito provavelmente pela pequena mudança que houve no peso dos pacientes durante o período de internação hospitalar.

Tabela 4 - Dados antropométricos, na internação e pré-alta hospitalar

\begin{tabular}{|c|c|c|c|}
\hline \multirow{2}{*}{ Variáveis } & Internação hospitalar & Pré-alta hospitalar & \multirow{2}{*}{$P$} \\
\hline & Média \pm DP & Média \pm DP & \\
\hline Peso (kg) & $73,0 \pm 18,5$ & $73,2 \pm 18,0$ & $0,203^{\star}$ \\
\hline IMC $\left(\mathrm{kg} / \mathrm{m}^{2}\right)$ & $27,3 \pm 6,2$ & $27,4 \pm 6,1$ & $0,192^{*}$ \\
\hline Classificação do IMC - n(\%) & & & $0,317^{\star *}$ \\
\hline Magreza $(<22$ kg/m²) & $13(21,0)$ & $13(21,0)$ & \\
\hline Eutrofia $\left(22-27 \mathrm{~kg} / \mathrm{m}^{2}\right)$ & $16(25,8)$ & $15(24,2)$ & \\
\hline Excesso de peso $\left(>27 \mathrm{~kg} / \mathrm{m}^{2}\right)$ & $33(53,2)$ & $34(54,8)$ & \\
\hline$\overline{\mathrm{CB}}$ & $29,4 \pm 5,5$ & $29,5 \pm 5,4$ & $0,059^{*}$ \\
\hline Classificação CB - n(\%) & & & $1,000^{\star *}$ \\
\hline Desnutrição (<P10) & $16(25,8)$ & $16(25,8)$ & \\
\hline Risco nutricional (P10 - P25) & $5(8,1)$ & $5(8,1)$ & \\
\hline Eutrofia (P25 - P75) & $28(45,2)$ & $28(45,2)$ & \\
\hline Risco de sobrepeso (P75 - P85) & $8(12,9)$ & $8(12,9)$ & \\
\hline Sobrepeso (P85 - P90) & $2(3,2)$ & $2(3,2)$ & \\
\hline Obesidade (>P90) & $3(4,8)$ & $3(4,8)$ & \\
\hline$\overline{\mathrm{CP}}$ & $35,1 \pm 4,3$ & $35,2 \pm 4,2$ & $0,088^{*}$ \\
\hline Classificação da CP - n(\%) & & & $1,000^{* *}$ \\
\hline Risco de desnutrição (<31cm) & $8(13,1)$ & $7(11,5)$ & \\
\hline Adequado ( $\geq 31 \mathrm{~cm})$ & $53(86,9)$ & $54(88,5)$ & \\
\hline
\end{tabular}

Fonte: primária.

Nota: $\mathrm{DP}=$ desvio padrão; $\mathrm{p}=$ percentil $\mathrm{kg}=$ quilos; $\mathrm{IMC}=$ índice de massa corporal; $\mathrm{kg} / \mathrm{m}^{2}=$ quilos por metro quadrado; $\mathrm{n}=$ número; $\%=$ percentual $<=$ menor do que; >= maior do que; $\mathrm{CB}=$ circunferência do braço; $\mathrm{CP}=$ circunferência da panturrilha; $\geq=$ maior ou igual; $\mathrm{cm}=$ centímetro.

* teste $t$-student para amostras pareadas; ** teste Qui-Quadrado de McNemar.

\section{Discussão}

No presente estudo avaliou-se o estado nutricional e o consumo alimentar de idosos durante o período de internação hospitalar.

Quanto às características dos idosos avaliados, a idade média foi de $73,5 \pm 9,0$ anos, sendo que a maioria era do gênero feminino, 51,6\%. Estudo semelhante foi publicado por Almeida et al. (2008) com média de idade de $72 \pm 8$ anos, sendo que para este estudo também prevaleceu as internações de pacientes do gênero feminino, representando um total de $54 \%$ da amostra. Para Prates e Silva (2013), 
$60 \%$ dos avaliados eram do sexo feminino, sendo a idade média da amostra $75,7 \pm 13,3$ anos. Trabalhos publicados por Zanchim, Liberali e Coutinho (2013) e Azevedo et al. (2007) revelaram um predomínio de internações de pacientes do gênero masculino, contrariando os estudos citados anteriormente, porém, com idade média semelhantes.

Em estudos avaliando pacientes hospitalizados é comum encontrar algum tipo de desequilíbrio corporal e patologias que pode estar refletindo no estado nutricional (risco de desnutrição ou sobrepeso/obesidade). Dentre as doenças avaliadas no presente estudo, a maioria dos idosos apresentava problemas respiratórios $(62,9 \%)$, seguido de Hipertensão Arterial Sistêmica (HAS). Estudos publicados por Garcia, Merhi e Pereira, (2004), Azevedo et al. (2007), Almeida et al. (2008) e Santos (2007) encontraram um percentual inferior de pacientes com Doenças Respiratórias, 20,6\%, 13,12\%, $16,6 \%$ e $53,7 \%$, respectivamente. Em relação à Hipertensão Arterial Sistêmica (HAS), no presente estudo, foi encontrado um percentual de $24,2 \%$. Ao se examinar vários outros estudos, os mesmos comprovam elevados índices de HAS em idosos hospitalizados, como o estudo de Santos (2007), que relatou um percentual de 48,7\%, Prates e Silva (2013), com 85\% de hipertensos e Malta, Papini e Corrente (2013) publicaram que $74 \%$ dos idosos apresentavam HAS, seguido de outras doenças crônicas, como a dislipidemia e o diabetes melittus (DM).

Como descrito por Nunes (2004), as duas principais e mais frequentes causas de internação em idosos são a insuficiência cardíaca e coronariana e, em segundo lugar, as doenças pulmonares. O autor, também apontou que entre as causas intermediárias, estavam as crises hipertensivas, em ambos os gêneros. Porém a pesquisa de Gritti et al. (2015) revelou que a HAS e o DM foram as principais Doenças Crônicas Não Transmissíveis (DCNT) diagnosticadas nas reinternações da população estudada. Da mesma forma, Otero et al (2002) ressalta que nos últimos anos, ocorreram mudanças no perfil de saúde da população, por consequência, as doenças crônicas têm demonstrado maior predomínio.

Em relação ao tipo de dieta prescrita durante a internação hospitalar, neste estudo, a maioria dos pacientes avaliados estava com alimentação livre, seguido de hipossódica e branda. Este resultado foi parecido com os citados por Garcia, Merhi e Pereira (2004), Merhi et al. (2015), Prieto et al. (2006) e Gaino, Merhi e Oliveira (2007), nos quais também prevaleceu a prescrição da dieta livre e, em sequência, as dietas modificadas e branda. A dieta hospitalar contribui para o aporte de nutrientes ao paciente hospitalizado, oferecendo a este a possibilidade de preservar seu estado nutricional, bem como colabora no controle de doenças crônicas e agudas, sendo que a mesma desempenha um papel importante na experiência de internação (CORREIA et al., 2013).

Neste estudo, verificou-se, também, aumento significativo do consumo alimentar da internação para um dia pré-alta hospitalar em praticamente todos 
os nutrientes, exceto para o percentual de proteínas, que não foi significativo, e, para o percentual de lipídeos, em que houve redução significativa na segunda avaliação. Além disso, houve também, aumento significativo em relação à quantidade de refeições completas e à ingestão de frutas, verduras e líquidos durante o dia.

Esses resultados são positivos considerando que, mesmo não tendo diferenças significativas, alguns idosos que estavam em risco nutricional se alimentaram melhor do que no momento da internação. Alguns estudiosos, preocupados com a alimentação oferecida aos pacientes internados nos hospitais, desenvolveram diversas pesquisas para obterem resultados concretos sobre o assunto. Pode-se citar o estudo publicado por Kondrup et al. (2002), no qual avaliaram pacientes internados por um período superior a uma semana e verificaram que $25 \%$ dos pacientes apresentavam ingestão alimentar que atingia de 75 a 99\% das necessidades energéticas corporais. Por outro viés, o estudo de Barton et al. (2000) mostrou que a dieta hospitalar de rotina fornecia $2000 \mathrm{kcal} / \mathrm{dia}$, atendendo às necessidades nutricionais dos pacientes. Também observaram que mais de $40 \%$ da alimentação oferecida era desperdiçada, devido à baixa ingestão alimentar, resultando no consumo inadequado em relação às necessidades nutricionais, o que poderia justificar a perda de peso em muitos pacientes no período de internação hospitalar. Em vista dos argumentos apresentados, percebeu-se que, a alimentação ofere- cida pelos hospitais é de boa qualidade, sendo suficiente para suprir as necessidades nutricionais dos pacientes, e que depende apenas da condição do paciente aceitar a alimentação oferecida.

No presente estudo, houve aumento significativo da ingestão de líquidos no período de internação hospitalar, quando comparado com a ingestão no domicílio. $\mathrm{O}$ aumento se deu principalmente nos pacientes que ingeriam baixa quantidade de água ( $<$ de 3 xícaras por dia). Dos cinco pacientes que ingeriam $<3$ xícaras de líquidos/dia, quatro deles aumentaram a ingestão hídrica para 3-5 xícaras por dia e apenas um progrediu a ingestão para > de 5 xícaras de líquidos/dia. Carvalho e Zanardo (2010) avaliaram o consumo de água e outros líquidos em adultos e idosos, constatando consumo de 5,7 copos de água/dia e 9,1 copos de outros líquidos no decorrer do dia. Em um estudo publicado por Sass e Marcon (2012), encontrou-se baixa ingestão de água em idosos hospitalizados. O consumo de dois a três copos de água/dia foi evidenciado em $69,2 \%$ dos idosos dependentes e um percentual de $29,0 \%$ em idosos independentes. Os autores também citaram que apenas $15,4 \%$ dos idosos dependentes ingeriam mais de oito copos de água/dia e dos idosos independentes, apenas $19,4 \%$ ingeriam mais de oito copos de água/dia. De acordo com Sandri, Bernardi e Siviero (2012) apenas 13,3\% das mulheres avaliadas atingiram as recomendações nutricionais das Dietary Reference Intakes (DRIS), com ingestão média de $1805 \pm 897 \mathrm{ml} /$ dia de líquidos por dia. Tais dados apontam que, neste 
estudo, apesar da melhoria, a ingestão de líquidos, ainda está aquém do recomendado. O Ministério da Saúde preconiza para os idosos, a ingestão mínima de 2 litros (6 a 8 copos de água por dia).

Em relação ao consumo de fibras, no presente estudo, a população avaliada apresentou baixa ingestão na pré-hospitalização, havendo melhora de $(\mathrm{p}<0,001)$ na ingestão para a alimentação oferecida no hospital. Mesmo havendo esse aumento na ingestão de fibras de $9,6 \mathrm{~g} / \mathrm{dia}$, os resultados encontrados são inferiores ao recomendado para idosos. Valores superiores foram encontrados por Müller, Wichmann e Ohlweiler (2007) em que $73,8 \%$ da população avaliada apresentou uma ingestão média de $16,84 \pm 8,87 \mathrm{~g} /$ dia e sua variação foi de $3,99 \mathrm{~g}$ a $59,06 \mathrm{~g}$. Os autores destacam que a média é considerada baixa, quando comparado à recomendação das fibras na prevenção das doenças cardiovasculares (DCVs). Sandri, Bernardi e Siviero (2012) encontraram que das 60 mulheres adultas e idosas avaliadas, apenas $46,7 \%$ das idosas atingiram as recomendações da ingestão de fibras pelas DRIS (21g/dia) e 35\% atingiram as recomendações conforme preconização do Guia Alimentar (25g/dia).

Resultado bastante positivo foi a diminuição da ingestão de lipídeos na dieta durante o período de internação. Isso ocorreu devido às mudanças nos hábitos alimentares, visto que, no hospital, a dieta oferecida era balanceada e variada, sendo preparada com reduzida quantidade de gordura, nos mais diversificados tipos de preparações, como grelhados, assados e refogados, não sendo oferecidas frituras. Comparando o recordatório alimentar de 24 horas pré-internação, com o pós-internação, verificou-se que, anterior à internação hospitalar, os idosos ingeriam quantidades superiores de lipídeos. Valores que se encontram dentro dos limites preconizados pelas Dietary Reference Intakes do Institute of Medicine (DRIS/IOM, 2010) (20-35\%). Em estudo publicado por Kucera, Siviero e Bonatto (2012) o consumo médio de lipídeos totais na dieta era de 19,63 $\pm 10,74 \%$. Esse estudo cita também que apenas $8,9 \%$ consumiam o macronutriente acima do recomendado e $15,6 \%$ estavam com ingestão adequada.

Percebeu-se também aumento significativo na ingestão de cálcio $(\mathrm{p}=0,012) \mathrm{e}$ de ferro $(p<0,001)$ na alimentação, mas que, mesmo assim, apenas a ingestão de ferro atingiu o consumo médio diário recomendado para os idosos. Estudos realizados por Malta, Papini e Corrente (2013) e Menezes, Nunes e Holanda (2005) observaram ingestão insuficiente do micronutriente cálcio em suas pesquisas. Dados controversos foram encontrados por Dobner, Blasi e Kirsten (2012) que encontraram adequada ingestão de cálcio e uma boa porcentagem de adequação do micronutriente ferro na alimentação dos idosos.

Conforme os resultados do estado nutricional dos idosos aplicando a MAN, o presente estudo demonstrou que prevaleceu a classificação de pacientes fora de risco, seguido de risco de desnutrição e em menor percentual a desnutrição. Resultados parecidos com prevalência 
de eutrofia foram encontrados por Colembergue e Conde (2011), com 64,3\% de eutróficos e Paz, Fazzio e Santos (2012) com 66,7\% de eutróficos. Dados controversos foram encontrados por Zanchim, Liberali e Coutinho (2013), em que $7,01 \%$ das mulheres eram eutróficas, 40,34\% em risco de desnutrição e 33,33\% desnutridas. Em relação aos homens, o risco de desnutrição foi maior, tendo um resultado de $67,14 \%$, seguido de $21,91 \%$ de desnutrição e $10,95 \%$ de eutrofia.

Os resultados mostraram que não houve mudanças significativas nos dados antropométricos, especialmente, quase não se observou alterações no peso, as mínimas alterações foram de $50 \mathrm{~g}$ a $900 \mathrm{~g}$ nos pacientes que perderam peso e de $100 \mathrm{~g}$ a $4,8 \mathrm{~kg}$ nos pacientes que ganharam peso. Dentre os avaliados 29 $(46,77 \%)$ pacientes perderam peso, 24 $(38,71 \%)$ ganharam peso e $9(14,52 \%)$ mantiveram o peso. Estudo parecido foi publicado por Garcia, Merhi e Pereira (2004), em que os autores apontaram que a alteração de peso durante a internação expressa o impacto desta sobre $o$ estado nutricional, sendo que $43,8 \%$ dos pacientes perderam peso, sobretudo na faixa de 2,0 e $0,5 \mathrm{~kg} ; 19,4 \%$ mantiveram o peso, $11,8 \%$ ganharam peso e em $25,1 \%$ dos pacientes, não foi avaliada a variação de peso. Rezende et al. (2004), em seu estudo, relataram que a desnutrição hospitalar pode progredir à medida que aumenta o período de internação, por consequência, $46 \%$ dos pacientes já estavam desnutridos nos primeiros cinco dias. O número se elevou para $68 \%$ entre cinco e quinze dias e aumentou para $83 \%$ nos doentes internados há mais de quinze dias. Prieto et al. (2006), por sua vez, constataram que apesar de não ocorrer alterações no estado nutricional dos pacientes durante o período de internação hospitalar, avaliados por meio do IMC, houve redução significativa na média do peso corpóreo (74,0 vs $69,2 \mathrm{~kg}$ ). Os autores citam, também, que a alteração de peso foi em $10,3 \%$ da população em estudo, sendo que 2,4\% apresentaram perda superior a $1 \mathrm{~kg}$. Relacionam essa diminuição de peso com os processos mórbidos e um pior prognóstico clínico e nutricional dos pacientes. Levando-se em consideração os fatos argumentados, no presente estudo, as mínimas alterações de peso podem estar atribuídas ao tempo de internação, que variou de cinco até dez dias. Provavelmente, se os avaliados mantivessem em casa o mesmo padrão alimentar, haveria alterações nas proporções corporais. Outro aspecto que, provavelmente, influenciou o estado nutricional são as patologias de base, neste estudo, a maioria dos problemas de saúde relatados entre os avaliados, foram as doenças respiratórias, de um modo geral. Essas estão associadas ao aumento da taxa de metabolismo de repouso, devido à intensa atividade dos músculos respiratórios acessórios. Além, disso é conhecido que dentre os aspectos que auxiliam para o ganho ou a redução de peso corporal, é necessário uma regularidade em alimentar-se de forma equilibrada, incluindo todos os grupos alimentares de acordo com as necessidades individuais. 
Vários estudos relatam sobre o período de internação de idosos em hospitais, dentre eles: Merhi et al. (2015), que verificaram que o tempo de internação foi de 8,9 96,5 dias (mediana 7 dias); Gaino, Merhi e Oliveira (2007) que encontraram uma permanência por até três dias em $73 \%$ dos pacientes, $15 \%$ por um período de 4 a 6 dias, $5,5 \%$ de 7 a 9 dias e $5,5 \%$ permaneceram por um período $\geq$ a dez dias, e o estudo de Almeida et al. (2008) que publicaram uma média de permanência no hospital de 14 dias em pacientes com nutrição desiquilibrada, com ingestão alimentar inferior às necessidades corporais, sendo que os pacientes com padrão respiratório ineficaz permaneceram hospitalizados em média de 15 dias. Dessa forma, o tempo de permanência hospitalar é muito afetado pela condição de saúde/doença do idoso.

$\mathrm{O}$ presente estudo encontrou que, dos 62 idosos avaliados, $21 \%$ apresentavam magreza. Esse resultado é parecido com os publicados por Gaino, Merhi e Oliveira (2007), Sonsin, Bonfim e Silvia (2009) e Garcia, Merhi e Pereira (2004), que encontraram em seus estudos, respectivamente, $22 \%, 43,3 \%$ e $23,1 \%$ de pacientes idosos desnutridos. Sarni et al. (2005) ressaltam que é importante conhecer o estado nutricional dos pacientes, em virtude das evidências de que a desnutrição está associada a risco de infecções e a várias complicações metabólicas, aumentando o tempo de internação, os custos hospitalares e a morbimortalidade do indivíduo hospitalizado. Os autores citam também que a ausência do diagnóstico nutricional, implica numa terapia nutricional inadequada, que por consequência pode resultar em várias complicações, podendo levar ao óbito.

A desnutrição não é a única forma de classificação do estado nutricional dos idosos. No presente estudo, verificou-se que $53,2 \%$ dos idosos internados estavam com excesso de peso. O excesso de peso no indivíduo idoso se destaca em vários outros estudos, dentre eles: Fares et al. (2012), Prates e Silva (2013), Prieto et al. (2006), Adami, Feil e Bosco (2015), sendo que os últimos classificaram o estado nutricional dos idosos, segundo a Organização Pan-Americana de Saúde (OPAS), e encontraram que $43,6 \%$ dos avaliados estavam com sobrepeso e obesidade.

Pinheiro, Freitas e Corso (2004) comentam que o excesso de peso vem se desencadeando na população em geral devido às mudanças epidemiológicas e a transição nutricional, considerando que ocorreu um aumento do consumo de alimentos com alta densidade energética e baixa ingestão de fibras.

Segundo o Guia Alimentar para a população Brasileira (2014), a tendência mundial de evolução da desnutrição tem decaído. Embora ainda existam grandes variações entre os países, o problema persiste com magnitude importante em países menos desenvolvidos. No Brasil, nos últimos anos, ocorreu um declínio significativo do número de idosos desnutridos, isso se deu devido às políticas públicas bem sucedidas de distribuição de renda, de erradicação da pobreza absoluta e de ampliação do acesso a serviços básicos de saúde, saneamento e educação. De acordo com o Instituto Bra- 
sileiro de Geografia e Estatística (2010), em resultados de pesquisas nacionais, conclui-se que os dados antropométricos são indicativos de que o sobrepeso e a obesidade estão presentes em todas as faixas etárias, sendo um problema de grande relevância para a saúde pública no país.

Outro aspecto que se deve considerar que, com o processo do envelhecimento algumas alterações estruturais e funcionais podem ocorrer, entre as quais a perda progressiva da massa corpórea magra e modificações no padrão de distribuição da gordura corporal, no qual o tecido adiposo dos braços e das pernas diminui, aumentando o acúmulo de gordura na região do tronco (MENEZES; MARUCCI, 2005).

Dentre as limitações do presente estudo, destaca-se que não se atingiu a amostra prevista do projeto, não permitindo generalizações externas, pois um dos critérios de inclusão era permanecer internado no mínimo cinco dias, alguns pacientes receberam alta antes deste período. Outro fato foi que alguns completavam o período de cinco dias de internação e recebiam alta no final de semana. Também se pode destacar que o período determinado não foi o suficiente para alcançar o tamanho da amostra, pelo motivo de o hospital ser de pequeno porte e, portanto, com menos atendimentos do que o estimado previamente. Um ponto forte do estudo foi a aplicação dos recordatórios alimentares de $24 \mathrm{~h}$, em que questionava-se ao paciente, levando esse a relatar o que havia consumido no dia anterior, com suas correspondentes porções, apesar de os avaliados não terem alterado a composição corporal.

\section{Conclusão}

Os resultados obtidos neste estudo mostraram elevada prevalência de excesso de peso na população estudada. Observou-se que não ocorreu perda de peso significativa nos dias de internação hospitalar e houve melhora significativa na ingestão alimentar para calorias e para a maioria dos nutrientes. Assim, sugere-se que houve melhora na qualidade das refeições consumidas, sendo essas ofertadas em horários determinados pelo próprio hospital, com cardápios elaborados pela nutricionista, satisfazendo às necessidades energéticas do organismo. Conclui-se dessa forma, que o diagnóstico nutricional do idoso hospitalizado é fundamental, para melhor manejo dos cuidados dietéticos. Ressalta-se que outros estudos se fazem necessários para conhecer e avaliar o real estado nutricional dos idosos durante o período de internação hospitalar e seu percurso em relação à recuperação da saúde.

\section{Monitoring of nutritional status and food intake of elderly during the hospitalization}

\section{Abstract}

The objective of this study was to determine the nutritional status and elderly dietary intake during the hospitalization period in a hospital in Guaporé, Rio Grande do Sul. It is about a cohort study, with primary data 
collection, conducted from August 2014 to July 2015. The sample was 62 elderly. The data information were: gender, age, disease and the type of diet. It was applied the Mini Nutritional Assessment (MNA) and the 24Hour Dietary Recall (24HR), which was evaluated the total calories, carbohydrate, protein, fat, fiber, calcium and iron. Both of them were done at the admission, in 24 hours, and later at hospital discharge. Statistical analysis was performed with the Statistical Package for Social Sciences version 21.0. Quantitative variables were expressed as average and standard deviation or median and interquartile range and quality by absolute and relative frequencies. The results show that $21 \%$ of seniors were thinness, $25.8 \%$ showed normal weight and $53.2 \%$ were overweight, and there were not significant changes about anthropometric measurements during the hospitalization. There was a significant increase in food intake from hospitalization to pre-discharge day in all nutrients except for the percentage of proteins and the lipids, where there was a significant reduction in the second evaluation. It was found a high prevalence of overweight in the sample, no weight loss in the days of hospitalization, but with better food intake for calories and most of the nutrients.

Keywords: Nutritional Status. Hospitalization period. Food intake. Elderly.

\section{Referências}

ADAMI, F. S.; FEIL, C. C.; BOSCO, S. D. Estado nutricional relacionado à qualidade de vida em idosos. Revista Brasileira de Ciências do Envelhecimento Humano, Passo Fundo, v. 12, n. 1, p. 28-40, jan./abr. 2015.

ALMEIDA, M. A. et al. Diagnósticos de enfermagem e intervenções prevalentes no cuidado ao idoso hospitalizado. Revista
Latino-Americana de Enfermagem, Ribeirão Preto, v. 16, n. 4, [s. p.], jul./ago. 2008.

AMERICAN HEART ASSOCIATION. National Center For Health Statistics. NHANES III. Hyattsville: Public Health Service, 1996.

AQUINO, R. C. Fatores associados ao risco de desnutrição e desenvolvimento de instrumentos de triagem nutricional. Tese (Doutorado em saúde Pública) - Faculdade de Saúde Pública, Universidade de São Paulo, São Paulo, 2005.

AZEVEDO, L. C. et al. Principais fatores da mini avaliação nutricional associada a alterações nutricionais de idosos hospitalizados. Arquivos Catarinenses de Medicina, Florianópolis, v. 36, n. 3, p. 7-14, 2007.

BARTON, A. D. et al. High food wastage and low nutritional intakes in hospital patients. Clinical Nutrition, v. 19, n. 6, p. 445-449, 2000.

BRASIL. Ministério da Saúde. Secretaria de Atenção à Saúde. Departamento de Atenção Básica. Guia alimentar para a população. 2. ed. Brasília, DF: Ministério da Saúde, 2014. Disponível em: <http://portalsaude. saude.gov.br/images/pdf/2014/novembro/05/ Guia-Alimentar-para-a-pop-brasiliera-Miolo-PDF-Internet.pdf>. Acesso em: 16 nov. 2016.

CARVALHO, A. P. L.; ZANARDO, V. P. S. Consumo de água e outros líquidos em adultos e idosos residentes no município de Erechim - Rio Grande do Sul. Perspectiva, Erechim, v. 34, n. 125, p. 117-126, mar. 2010.

COLEMBERGUE, J. P.; CONDE, S. R. Uso da mini avaliação nutricional em idosos institucionalizados. Scientia Medica, Porto Alegre, v. 21, n. 2, p. 59-63, 2011.

CORREIA, M. G. S. et al. A importância da dieta hospitalar na recuperação de pacientes diabéticos. Cadernos de Graduação - Ciências Biológicas e da Saúde, Aracaju, v.1, n.16, p.47-56, mar. 2013. 
CRESTANI, N. et al. Perfil nutricional de pacientes adultos e idosos admitidos em um hospital universitário. Revista Ciência \& Saúde, Porto Alegre, v. 4, n. 2, p. 45-49, jul./ dez. 2011.

DOBNER, T.; BLASI, T. C.; KIRSTEN, V. R. Perfil nutricional de idosos residentes em instituição geriátrica no interior do RS. Revista Brasileira de Ciências do Envelhecimento Humano, Passo Fundo, v. 9, n. 1, p. 109-118, jan./abr. 2012.

FARES, D. et al. Fatores associados ao estado nutricional de idosos de duas regiões do Brasil. Revista da Associação Médica Brasileira, São Paulo, v. 58, n. 4, p. 434-441, jul./ago. 2012.

FÉLIX, L. N.; SOUZA, E. M. T. Avaliação nutricional de idosos em uma instituição por diferentes instrumentos. Revista de Nutrição, Campinas, v. 22, n. 4, p. 571-580, jul./ago. 2009.

FRISANCHO, A. R. Anthropometric standars for the assessment of growth and nutritional status. Published in the United States of America by. The University of Michigan Press. Manufactured in the United States of America. University of Michigan, 1990.

GAINO, N. M.; MERHI, V. A. L.; OLIVEIRA, M. R. M. Idosos hospitalizados: estado nutricional, dieta, doença e tempo de internação. Revista Brasileira de Nutrição Clínica, Porto Alegre, v. 22, n. 4, p. 273-279, 2007.

GARCIA, R. W. D. A dieta hospitalar na perspectiva dos sujeitos envolvidos em sua produção e em seu planejamento. Revista de Nutrição, Campinas, v. 19, n. 2, p. 129-144. mar./abr. 2006.

GARCIA, R. W. D.; MERHI, V. A. L.; PEREIRA, A. M. Estado nutricional e sua evolução em pacientes internados em clinica médica. Revista Brasileira de Nutrição Clínica, Porto Alegre, v. 19, n. 2, p. 59-63, abr.jun. 2004.

GRITTI, C. C. et al. Doenças crônicas não transmissíveis e antecedentes pessoais em reinternados e contribuição da terapia ocu- pacional. Cadernos Saúde Coletiva, Rio de Janeiro, v. 23, n. 2, p. 214-219, 2015.

HORNBY, S. T. et al. Relationship between structural and functional measures of nutritional status in a normally nourished populations. Clinical Nutrition, v. 24, n. 3, p. 421-426, June 2005 .

INSTITUTO BRASILEIRO DE GEOGRAFIA E ESTATÍSTICA. Pesquisa de orçamentos familiares 2008-2009: antropometria e estado nutricional de crianças e adolescentes e adultos no Brasil. Rio de Janeiro: IBGE, 2010.

LIPSCHITZ, D. A. Screening for nutritional status in the elderly. Primary Care, v. 21, n. 1, p. 55-67, 1994.

KAMIMURA, M. A. et al. Avaliação nutricional. In: CUPPARI, L. Guias de nutrição: nutrição clínica no adulto. Barueri, SP: Manole, 2. ed. p. 89-127, 2005.

KONDRUP, J. et al. Incidence of nutritional risk and causes of inadequate nutritional care in hospitals. Clinical Nutrition, v. 21, n. 6, p. 461-468, Dec. 2002.

KRUIZENGA, H. M. et al. Effectiveness and cost-effectiveness of early screening and treatment of malnourished patients.The American Journal of Clinical Nutrition, v. 82, n. 5, p. 1082-1089, Nov. 2005.

KUCERA, M. O. R.; SIVIERO, J.; BONATTO, S. Consumo de lipídeos e estado nutricional de idosos participantes do projeto NUTENV da Universidade de Caxias do Sul. Revista Brasileira de Ciências do Envelhecimento Humano, Passo Fundo, v. 9, n. 3, p. 426-438, set./dez. 2012.

MALTA, M. B.; PAPINI, S. J.; CORRENTE, J. E. Avaliação da alimentação de idosos de município paulista: aplicação do Índice de Alimentação Saudável. Ciência e Saúde Coletiva, Rio de Janeiro, v. 18, n. 2, fev. 2013.

MENEZES, T. N.; MARUCCI, M. F. N. Antropometria de idosos residentes em instituições geriátricas, Fortaleza, CE. Revista 
de Saúde Pública, São Paulo, v. 39, n. 2, p. 169-175, 2005.

MENEZES, T. N.; NUNES, M. F. M.; HOLANDA, I. M. M. Ingestão de cálcio e ferro alimentar por idosos residentes em instituições geriátricas de Fortaleza. Revista Saúde. com, v. 1, n. 2, p. 100-109, 2005.

MERHI, V. A. L. et al. Perda de peso hospitalar, dieta prescrita e aceitação de alimentos. Arquivos Brasileiros de Cirurgia Digestiva, São Paulo, v. 28, n. 1, p. 8-12, 2015. Disponível em: <http://www.scielo.br/pdf/abcd/v28n1/ pt_0102-6720-abcd-28-01-00008.pdf>. Acesso em: 16 nov. 2016.

MIRANDA, D. E .G. A. et al. Manual de avaliação nutricional do adulto e do idoso. Rio de Janeiro: Rubio, 2012.

MÜLLER, A. R.; WICHMANN, F. M. A.; OHLWEILER, Z. N. C. Perfil lipídico da dieta alimentar como fator de risco para doenças cardiovasculares em idosas ativas. Revista Brasileira de Geriatria e Gerontologia, Rio de Janeiro, v. 10, n. 2, p. 179-189, 2007.

NASCIMENTO, C. M. et al. Estado nutricional e fatores associados em idosos do Município de Viçosa, Minas Gerais, Brasil. Cadernos de Saúde Pública, Rio de Janeiro, v. 27, n. 12, p. 2409-2418, dez. 2011.

NORMAN, K. et al. Prognostic impact of disease-related malnutrition. Clinical $\mathrm{Nu}$ trition, v. 27, n. 1, p. 5-15, Feb. 2008.

NUNES, A. O envelhecimento populacional e as despesas do Sistema Único de Saúde. In: CAMARANO, A. A. (Org.). Os novos idosos brasileiros: muito além dos 60? 2. ed. Rio de Janeiro: IPEA, 2004. p. 427-450.

ORGANIZAÇÃO MUNDIAL DA SAÚDE. El estado físico: uso e interpretación de la antropometría. Genebra: OMS, 1995. (Serie de Informes Técnicos). p. 468. Disponível em: <http:/apps.who.int/iris/bitstream/10665/42132/1/WHO_TRS_854_spa.pdf>. Acesso em: 16 nov. 2016.
Informe Mundial sobre el envejecimiento y la salud. Genebra: WHO, 2015. Disponível em: <http://www.who.int/ageing/ publications/world-report-2015/es/>. Acesso em: 16 nov. 2016.

OTERO, U.B. et al. Mortalidade por desnutrição em idosos, região sudeste do Brasil, 1980-1997. Revista de Saúde Pública, São Paulo, v. 36, n. 2, p. 141-148, 2002.

PAGANOTTO, V; SILVEIRA, E. A; VELASCO, W. D. Perfil das hospitalizações e fatores associados em idosos usuários do SUS. Revista Ciência \& Saúde Coletiva, Rio de Janeiro, v. 18, n. 10, p. 3061-3070, mar. 2012.

PAZ, R. C.; FAZZIO, D. M. G.; SANTOS, A. L. B. Avaliação nutricional em idosos institucionalizados. Revisa 2012, v. 1, n. 1, p. 9-18, jan./jun. 2012. Disponível em: <http://revistafacesa.senaaires.com.br/index.php/revisa/ article/view/6/3>. Acesso em: 16 nov. 2016.

PINHEIRO, A. R. O.; FREITAS, S. F. T.; CORSO, A. C. T. Uma abordagem epidemiológica da obesidade. Revista de Nutrição, Campinas, v. 17, n. 4, out./dez. 2004.

PRATES, R. E.; SILVA, A. C. P. Avaliação do conhecimento nutricional e de hábitos alimentares de pacientes com doenças crônicas não transmissíveis em hospital particular no sul do Brasil. Revista da Associação Brasileira de Nutrição, São Paulo, v. 5, n. 1, p. 21-27, jan./jun. 2013.

PRIETO, B. D. et al. Intervenção nutricional de rotina em pacientes de um hospital privado. Revista Brasileira de Nutrição Clínica, Porto Alegre, v. 21, n. 3, p. 181-187, 2006.

REZENDE, I. F. B. et al. Prevalência da desnutrição hospitalar em pacientes internados em um hospital filantrópico em Salvador (BA), Brasil. Revista de Ciências Médicas e Biológicas, Salvador, v. 3, n. 2, p. 194-200, jul./dez. 2004. 
SANDRI, R.; BERNARDI, J. R.; SIVIERO, J. Consumo de fibras e líquidos em mulheres participantes de uma universidade da terceira idade no Sul do Brasil. Revista Brasileira de Ciências do Envelhecimento Humano, Passo Fundo, v. 9, n. 2, p. 213-225, maio/ ago. 2012.

SANTOS, M. I. P. O. Perfil dos idosos internados no Hospital Geral em Belém - Pará. Escola Anna Nery Revista de Enfermagem, Rio de Janeiro, v. 11, n. 1, mar. 2007.

SARNI, R. O. S. et al. Tratamento da desnutrição em crianças hospitalizadas em São Paulo. Revista da Associação Médica Brasileira, São Paulo, v. 51, n. 2, p. 106-112, mar./abr. 2005.

SASS, A.; MARCON, S. S. Dependência para alimentar-se e consumo alimentar em idosos hospitalizados. Revista Brasileira de Enfermagem, Brasília, v. 65, n. 6, nov./dez. 2012.

SILVA, J. L. et al. Fatores associados à desnutrição em idosos institucionalizados. Revista Brasileira de Geriatria e Gerontologia, Rio de Janeiro, v. 18, n. 2,p. 443-451, jun. 2015.

SONSIN, P. B.; BONFIM, C.; SILVIA, A. L. N. D. Análise da assistência nutricional a pacientes disfágicos hospitalizados na perspectiva de qualidade. Mundo Saúde, São Paulo, v. 33, n. 3, p. 310-319, 2009. Disponível em: <http://www.saocamilo-sp.br/pdf/ mundo_saude/69/310a319.pdf $>$. Acesso em: 15 nov. 2016.

VIDAL, A. et al. Prevalencia de malnutrición en los servicios médicos y quirúrgicos de un hospital universitário. Nutrición Hospitalaria, Madrid, v. 23, n. 3, mayo/jun. 2008.

WAITZBERG, D. L.; CAIEFFE, W. T.; CORREIA, M. E. T. D. Inquérito brasileiro de avaliação nutricional hospitalar (IBRANUTRI). Revista Brasileira de Nutrição Clínica, Porto Alegre, v. 14, p. 124-134, 1999.
ZANCHIM, M. C.; LIBERALI, R.; COUTINHO, V. Estado nutricional de idosos hospitalizados em um hospital geral de alta complexidade do Estado do Rio Grande do Sul. Revista Brasileira de Nutrição Clínica, Porto Alegre, v. 28, n. 4, out./dez. 2013. 